\title{
Numerical Simulation of Internal Flow Field, Electromagnetic Field and Temperature Field in Deep-Water Motor
}

\author{
Qi Zhang , Zengliang Li, Ran Yu, Zhaocheng Sun and Le Zhang \\ College of Mechanical Electronic Engineering, China University of Petroleum, Qingdao 266580 China
}

\begin{abstract}
In this paper, considering the heat exchange between the ends of stator\&rotor and lubricating oil, the internal flow field model is established to analyse the flow state. The variation law of the pressure drops in the internal flow field and the friction loss of the rotor surface are specified. The operation characteristic of the motor and the variation law of the magnetic field loss are obtained through the analysis of motor magnetic field. Based on the results of internal flow field and magnetic field, the temperature distribution law of motor is obtained. Compared with the result of the temperature simulation in which only air gap path is considered, the stator temperature shows totally different distribution law, and the stator temperature increases from $32{ }^{\circ} \mathrm{C}$ to $42^{\circ} \mathrm{C}$. Compared with the result of the temperature simulation without considering heat source loss change, the maximum temperature of the motor decreases from $71^{\circ} \mathrm{C}$ to $49^{\circ} \mathrm{C}$. The results show that the ends of stator\&rotor and the variation of heat source loss can influence the temperature simulation results.
\end{abstract}

\section{Introduction}

As oil filled asynchronous motors, Deep-water motors are not only meeting the requirement of power, but also have stronger ability to adapt complex work environment. With the development of marine equipment, the unit capacity of Deep-water motor is increasing. The motor get more loss, which makes the temperature rise. The service life of motor will be shortened as the motor is overheating. Due to the special working conditions, the maintenance cost of the motor is high. Accordingly, it is important to obtain the rules of motor temperature distribution accurately.

There are many analytical methods of the temperature field [1-5]. Finite volume method is widely used as a method of coupling flow field, magnetic field and temperature field [6-10]. The establishment of temperature field model and the calculation of heat source loss are the basis of numerical simulation. The traditional modelling approach only considers the influences of air gaps path on the temperature distribution, and ignores the influence of the end of stator\&rotor on temperature [11-12]. The calculation result of the heat source loss is always a constant, and ignores the influences of the temperature rise on viscosity and resistance [13-14]. These factors have resulted in the difference between thermal field simulation and actual temperature.

In this paper, the internal flow field, electromagnetic field and temperature field of the motor are established by using ANSYS. The variation law of heat source loss and the temperature distribution law of the motor are obtained.

\section{Internal flow field analysis of Deep- water motor}

\subsection{Internal flow field model}

According to the fluid dynamics theory, the governing equations of lubricating oil flow in the motor are mass conservation equation and momentum conservation equation.

$$
\partial u / \partial x+\partial v / \partial y+\partial w / \partial z=0
$$

$$
\begin{aligned}
& \frac{\partial\left(\rho u_{x}\right)}{\partial t}+\frac{\partial\left(\rho u_{x} u_{x}\right)}{\partial x}+\frac{\partial\left(\rho u_{y} u_{x}\right)}{\partial y}+\frac{\partial\left(\rho u_{z} u_{x}\right)}{\partial z}= \\
& \frac{\partial}{\partial x}\left(\mu \frac{\partial u_{x}}{\partial x}\right)+\frac{\partial}{\partial y}\left(\mu \frac{\partial u_{x}}{\partial y}\right)+\frac{\partial}{\partial z}\left(\mu \frac{\partial u_{x}}{\partial z}\right)-\frac{\partial p}{\partial x}+S_{u} \\
& \frac{\partial\left(\rho u_{y}\right)}{\partial t}+\frac{\partial\left(\rho u_{x} u_{y}\right)}{\partial x}+\frac{\partial\left(\rho u_{y} u_{y}\right)}{\partial y}+\frac{\partial\left(\rho u_{z} u_{y}\right)}{\partial z}= \\
& \frac{\partial}{\partial x}\left(\mu \frac{\partial u_{y}}{\partial x}\right)+\frac{\partial}{\partial y}\left(\mu \frac{\partial u_{y}}{\partial y}\right)+\frac{\partial}{\partial z}\left(\mu \frac{\partial u_{y}}{\partial z}\right)-\frac{\partial p}{\partial x}+S_{v}
\end{aligned}
$$




$$
\begin{aligned}
& \frac{\partial\left(\rho u_{z}\right)}{\partial t}+\frac{\partial\left(\rho u_{x} u_{z}\right)}{\partial x}+\frac{\partial\left(\rho u_{y} u_{z}\right)}{\partial y}+\frac{\partial\left(\rho u_{z} u_{z}\right)}{\partial z}= \\
& \frac{\partial}{\partial x}\left(\mu \frac{\partial u_{z}}{\partial x}\right)+\frac{\partial}{\partial y}\left(\mu \frac{\partial u_{z}}{\partial y}\right)+\frac{\partial}{\partial z}\left(\mu \frac{\partial u_{z}}{\partial z}\right)-\frac{\partial p}{\partial x}+S_{w}
\end{aligned}
$$

where, $p$ is the pressure on the fluid micro body. $\rho$ is fluid density. $u_{\mathrm{x}} 、 u_{\mathrm{y}}$ and $u_{\mathrm{z}}$ are the components of velocity vector $u$ in the direction of $x, y$ and $z$.

The basic parameters of the Deep-water motor studied in this paper are as follows. The load power is $60 \mathrm{~kW}$. The rated voltage is $1140 \mathrm{~V}$. The inner and outer diameters of stator are $120 \mathrm{~mm}$ and $260 \mathrm{~mm}$. The air gap length is $0.6 \mathrm{~mm}$. The core length is $300 \mathrm{~mm}$. The slot ratio of stator and rotor is $36 / 26$. The extreme logarithm is 2 . The winding form is single layer concentric type. The number of turns per slot is 35 . The rated flow rate and pressure of single-stage impeller are $1 \mathrm{~m}^{3} / \mathrm{h}$ and $0.2 \mathrm{MPa}$. Considering the heat exchange between the ends of stator\&rotor and lubricating oil, the internal flow field model of the motor is established based on the single tooth and single groove of stator\&rotor as shown in Fig. 1.

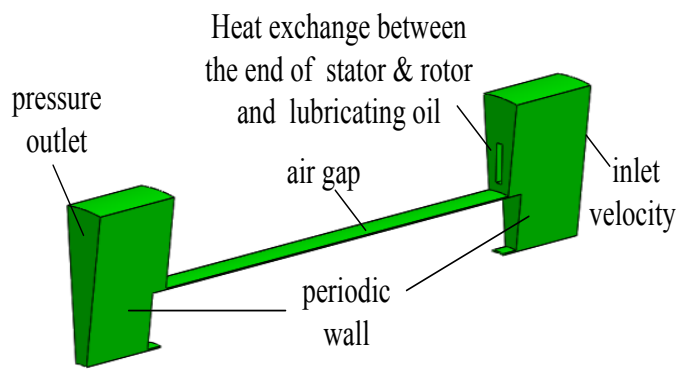

Figure 1. Internal flow field model.

\subsection{Internal flow field result analysis}

The boundary conditions of the internal flow field are set in Fig. 1. According to the rated flow of lubricating oil, inlet velocity is $0.004 \mathrm{~m} / \mathrm{s}$. The viscosity is $0.0971 \mathrm{~kg} / \mathrm{ms}$ at the temperature of $24^{\circ} \mathrm{C}$. The flow state in the air gap is laminar by Reynolds number. The rotor line speed is $9.19 \mathrm{~m} / \mathrm{s}$. The internal flow field center cross-section traces and radial velocity distribution of the air gap are obtained as shown in Fig.2. In the air gap, the lubricating oil flow is dominated by swirling flow, and it velocity distribution decreases from rotor side to stator side. The maximum speed is $9.2 \mathrm{~m} / \mathrm{s}$.

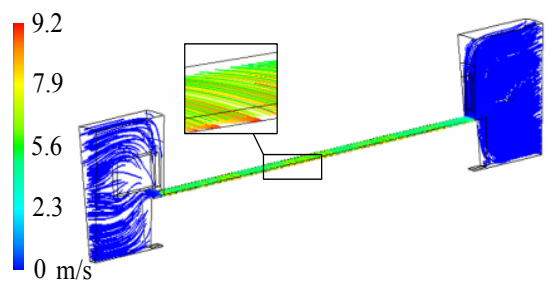

(a)Internal flow field center cross-section traces

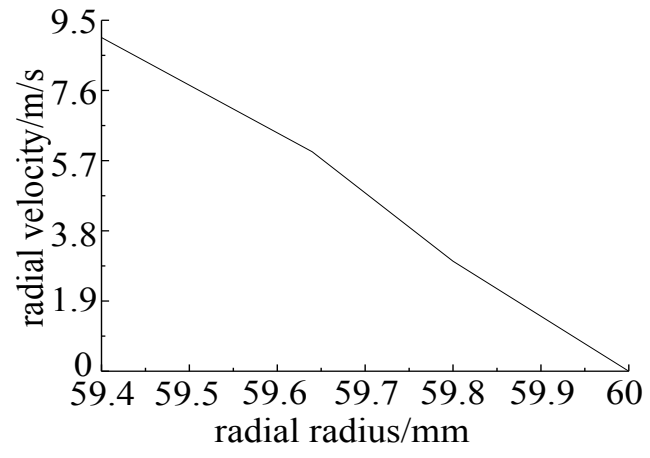

(b)Radial velocity distribution of the air gap

Figure 2. Internal flow field simulation result.

The motive power for lubricating oil flow comes from the impeller at the bottom of the motor. Considering the relationship between temperature and viscosity, the resistance of lubricating oil will change, which causes the impeller to provide pressure changes. According to the impeller characteristic curve, the change of pressure will make the corresponding flow change. In order to ensure the impeller work in the high-efficiency section, the pressure drop of the internal flow field under different viscosities and inlet velocities is given in Fig.3. As the viscosity and inlet velocity decrease, the pressure drop of the internal flow field decreases gradually. The results provide the basis for selecting the number of impellers in series.

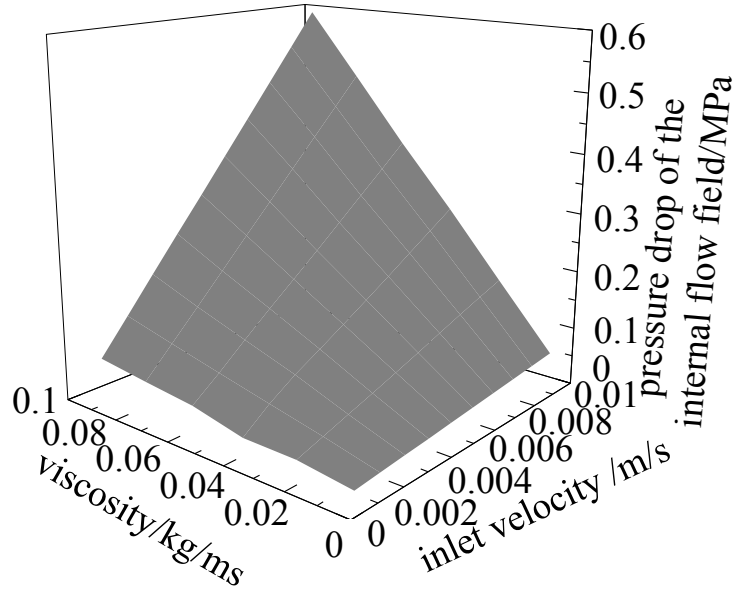

Figure 3. Pressure drop of the internal flow field under different viscosities and inlet velocities.

According to the analysis of the internal flow field, the friction loss of the rotor surface is related to the viscosity and the inlet velocity. As shown in Fig.4, the friction loss of the rotor surface increases with the increase of the viscosity, but basically unchanged with the increase of the inlet velocity. 


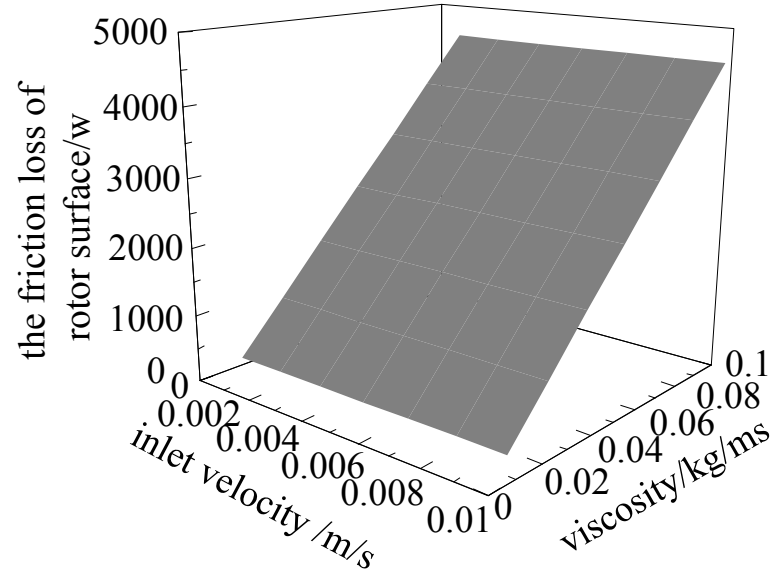

Figure 4. The friction loss of rotor surface under different viscosities and inlet velocities.

\section{Electromagnetic field analysis of Deep-water motor}

The Maxwell equation is used to represent the variation law of time varying electromagnetic field.

$$
\left\{\begin{array}{c}
\nabla \times H=J+\frac{\partial D}{\partial t} \\
\nabla \times E=-\frac{\partial B}{\partial t} \\
\nabla \cdot D=\eta \\
\nabla \cdot B=0
\end{array}\right.
$$

where, $H$ is the magnetic field strength. $B$ is magnetic induction intensity. $E$ is field strength. $D$ is electric displacement. $\mathrm{J}$ is current density. $\eta$ is charge density.

According to the size of stator and rotor, twodimensional electromagnetic field model is established as shown in Fig. 5.

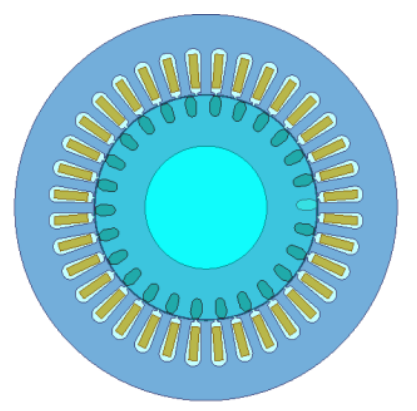

Figure 5. Two-dimensional electromagnetic field model.

The parallel boundary conditions of magnetic lines are applied on the outer circumference of stator. Constant power load is applied. Voltage source excitation is added. The simulation results of the electromagnetic field in the starting time of $0.2 \mathrm{~s}$ are obtained.

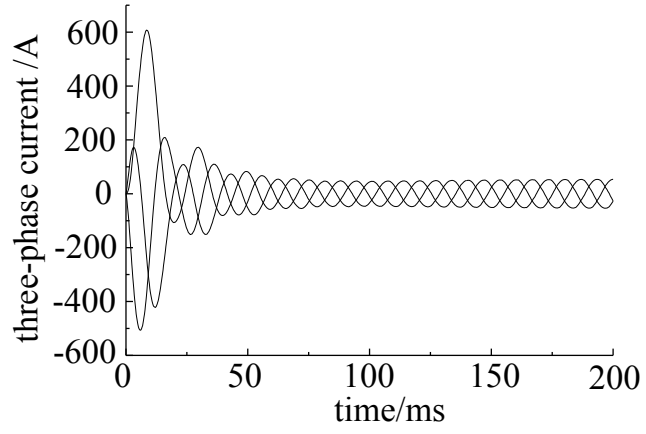

Figure 6. Three-phase current of the winding varies with time.

The three-phase current of the winding varies with time as shown in Fig.6. The three-phase current is sinusoidal alternating current. The current is very unstable at the initial time. The maximum amplitude is far more than the stable current. The stator winding current tends to be stable after $75 \mathrm{~ms}$.

The single-phase current changes with motor speed as shown in Fig. 7. When motor speed is 0 , the current is about 288A. With the increase of motor speed, the current decreases gradually. When the rated speed is reached, the current is about $33.6 \mathrm{~A}$.

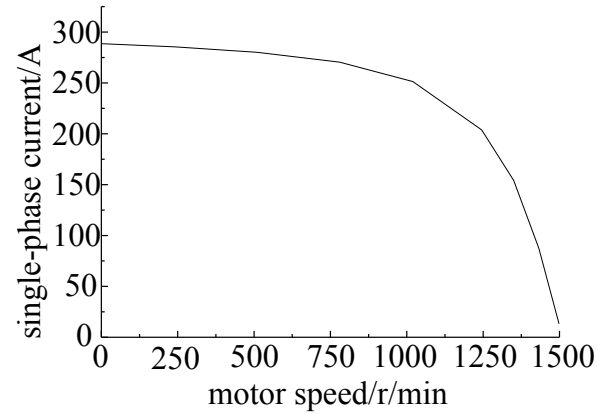

Figure 7. The single-phase current changes with motor speed.

The magnetic flux distribution in the motor at different times is shown in Fig.8. At 0.002s, the magnetic flux of rotor is mainly distributed on the surface of rotor tooth. The magnetic flux density of rotor yoke is small. At $0.01 \mathrm{~s}$, the motor operation gradually stabilized. The magnetic flux concentrated on the surface of rotor teeth gradually dispersed and transferred to rotor teeth. At $0.04 \mathrm{~s}$, the magnetic flux of rotor passes through the cogging, which is mainly distributed in the yoke, while almost no magnetic flux passes through rotor tooth surface.

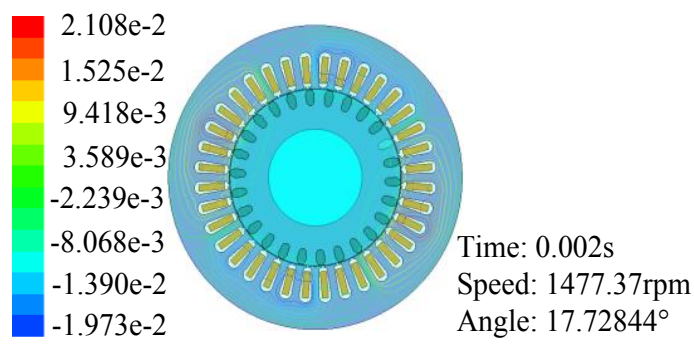

(a)Distribution of magnetic line at $0.002 \mathrm{~s}$ 


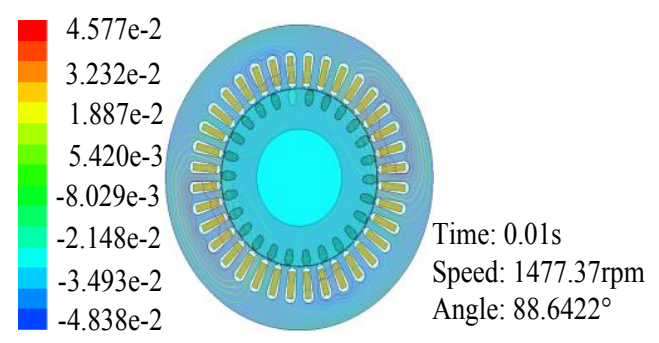

(b)Distribution of magnetic line at $0.01 \mathrm{~s}$

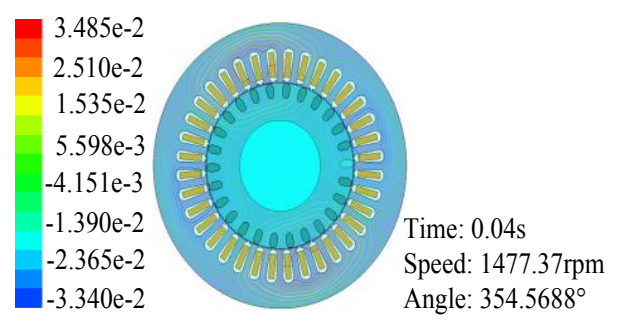

(c)Distribution of magnetic line at $0.04 \mathrm{~s}$

Figure 8. Magnetic flux distribution in the motor at different times.

According to the above analysis, the radial and tangential flux density curves of in different regions include tooth top, tooth and yoke in a stable power frequency period are shown in Fig.9. The radial and tangential flux density curves fluctuate obviously in the tooth top region. The flux density curve in the tooth region mainly fluctuates in the radial direction. In the yoke area, the radial flux density curve has less fluctuation, and the tangential flux density curve is smooth

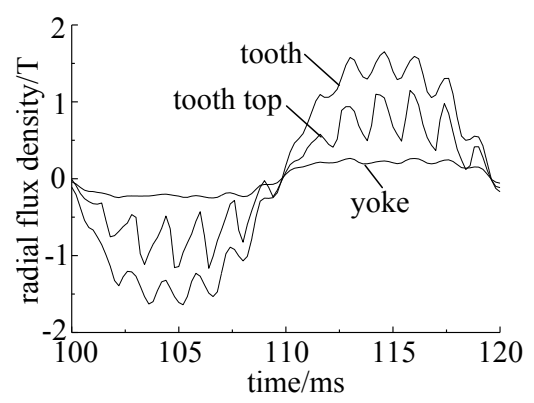

(a)Radial flux density curve

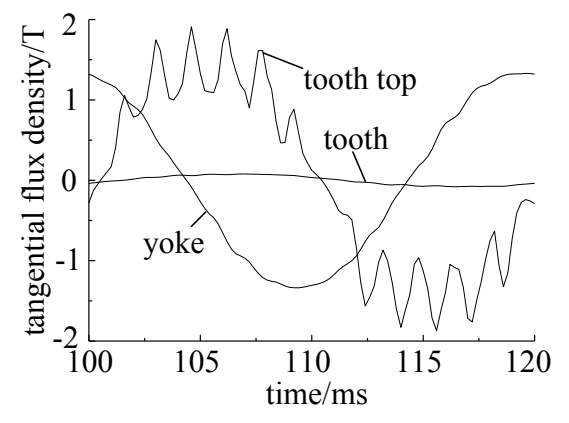

(b)Tangential flux density curve

Figure 9. Radial and tangential magnetic flux density curves in different regions.
In the actual motor magnetic field, due to the existence of harmonic magnetic field, it will make the radial and tangential magnetic fluxes produce nonpositive rotation changes. The magnetization density of harmonic number of different harmonic times is introduced and calculated by Fourier decomposition method.

$$
\begin{aligned}
& P=\sigma_{h} \sum_{k=1}^{N} f_{k}\left(\left(B_{r \max }\right)_{k}^{2}+\left(B_{t \max }\right)_{k}^{2}\right)+\sigma_{e} \sum_{k=1}^{N} f_{k}^{2}\left(\left(B_{r \max }\right)_{k}^{2}+\left(B_{t \max }\right)_{k}^{2}\right) \\
& +\sigma_{a} \sum_{k=1}^{N} f_{k}^{1.5}\left(\left(B_{r \max }\right)_{k}^{1.5}+\left(B_{t \max }\right)_{k}^{1.5}\right)
\end{aligned}
$$

where $\sigma_{\mathrm{h}}$ and $\alpha$ are the hysteresis loss coefficient. $\sigma_{\mathrm{e}}$ is the eddy current loss coefficient. $\sigma_{\mathrm{a}}$ is the abnormal loss coefficient. $B_{\text {rmax }}$ and $\mathrm{B}_{\text {tmax }}$ are the radial and tangential fluxes of the different harmonic times. $f_{k}$ is the different harmonic frequency.

The iron loss density distribution is obtained according to the equation (4) as shown in Fig. 10.

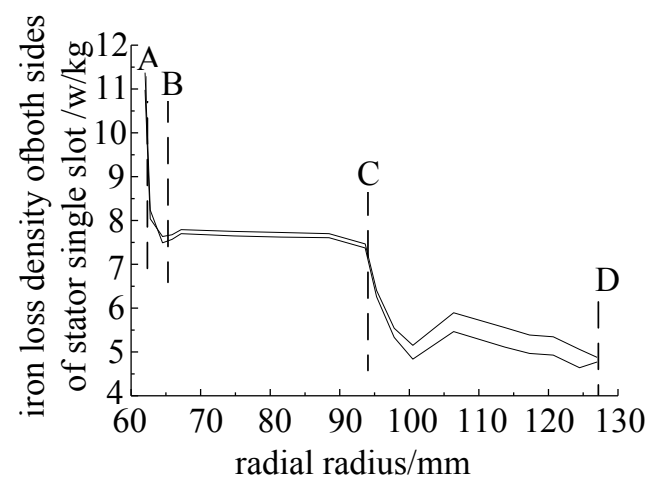

Figure 10. Iron loss density distribution.

In Fig. 10, $\mathrm{AB}$ is tooth top region. $\mathrm{BC}$ is tooth region. $\mathrm{CD}$ is yoke region. The distribution regularity of iron loss density both sides of stator single slot has the same trend. In the tooth top region, iron loss density decreases sharply with the increase of the radius. The iron loss density in the tooth region is basically unchanged. Iron loss density in the yoke region first decreases, then increases and then decreases with the increase of the radius, which is related to the direction of the magnetic field lines in the yoke.

The current loss in bar-conductor and winding is called as copper loss. It is calculated according to JouleLenz's law.

$$
P_{1}=3 I_{x}^{2} R_{x}
$$

where, $I_{\mathrm{x}}$ is the excitation current. $R_{\mathrm{x}}$ is the equivalent resistance converted to the reference operating temperature.

The change of copper loss under different viscosity is obtained by using viscosity instead of temperature. As shown in Fig.11, copper loss decreases with the increase of viscosity. 


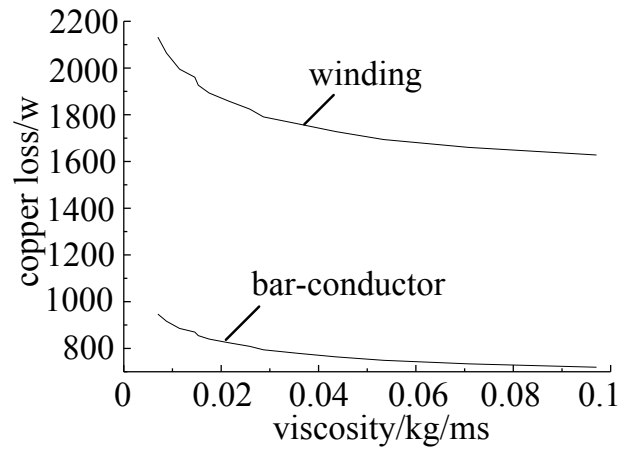

Figure 11. Variation law of copper loss.

\section{Temperature field analysis of Deep- water motor}

According to heat transfer theory, the fluid-solid coupling interface of motor has the same or the conservation of temperature $T$ and heat flux density $q$, and its expression is:

$$
\begin{aligned}
& T_{\text {soild }}=T_{\text {fluid }} \\
& q_{\text {soild }}=q_{\text {fluid }}
\end{aligned}
$$

The lubricating oil in the motor is given inlet temperature $T_{\mathrm{f}}$. The third boundary conditions of heat transfer are selected.

$$
\left(-\lambda \frac{\partial T}{\partial n}\right)_{\text {soild }}=\left(\alpha\left(T-T_{f}\right)\right)_{\text {fluid }}
$$

The heat transfer process in the fluid-solid coupling region is considered as a whole heat transfer process by the finite volume method.

The three-dimensional temperature field model of motor is established by using SolidWorks, which is shown in Fig. 12(a). The model is divided into 9 subblocks such as stator block and rotor block by using ICEM. On the basis of dividing the hexahedral grids in each sub-block, the sub-blocks are spliced as shown in Fig.12(b). The meshes of the interfaces between the subblocks should be consistent.

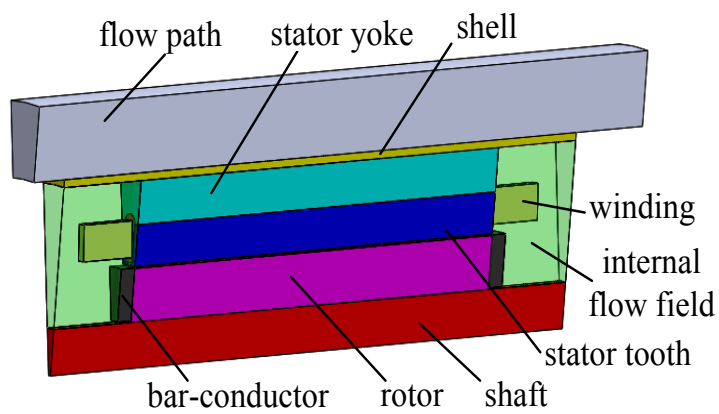

(a)Three-dimensional temperature field model

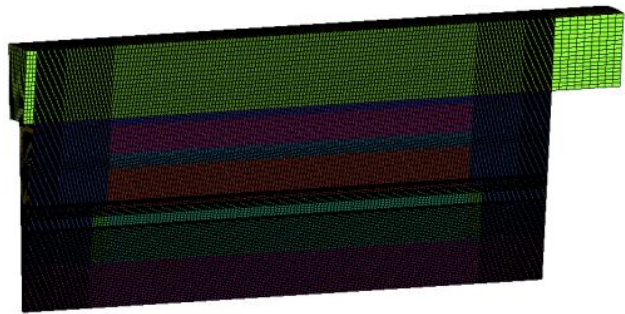

(b)Meshing

Figure 12. Three-dimensional temperature field model and meshing.

According to the analysis results of internal flow field and electromagnetic field, the UDF command is used to realize the variation law of rotor surface friction loss and copper loss with viscosity. The iron loss density is applied in different regions of the stator. The pressure inlet and the pressure outlet are set in the outer flow path. The other settings are consistent with the analysis of the internal flow field. The simulation results of the motor temperature are obtained as shown in Fig. 13.

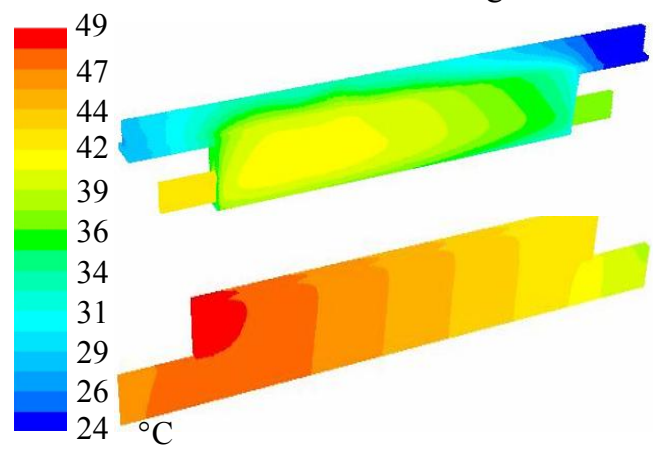

(a)Temperature distribution

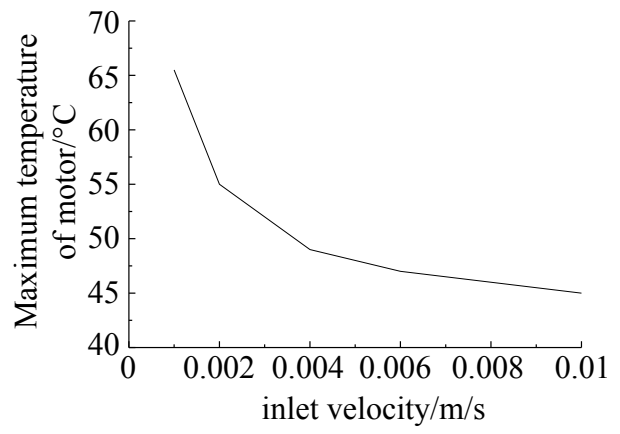

(b)Maximum temperature at different inlet velocities

Figure 13. Temperature field simulation result.

In Fig. 13, the rotor temperature is distributed along the axial direction, and the maximum temperature is $49^{\circ} \mathrm{C}$ at the air gap outlet because the rotor heat is mainly taken away by the air gap lubricating oil. Stator temperature is significantly lower than rotor temperature. Shell temperature is the lowest. The ends temperature of the stator and rotor is greater at the air gap outlet side than at the air gap inlet side. With the increase of the inlet velocity, the maximum temperature of the motor 
gradually decreases. The variation of the motor temperature after $0.004 \mathrm{~m} / \mathrm{s}$ is small.

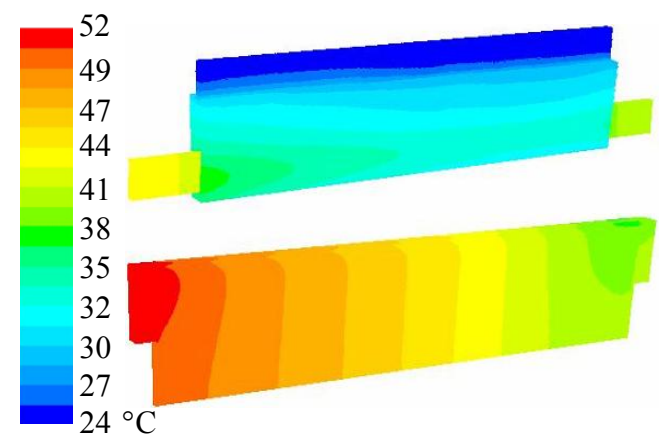

Figure 14. Temperature simulation considering only the air gap path.

Compared with the simulation results in Fig.13 and Fig. 14, the maximum temperature of the motor slightly increases. The tendency of the stator temperature is greatly different. This is mainly because the heating of the motor increases the temperature of the lubricating oil on the outlet side of the air gap. Due to the heat exchange between the end of the stator and rotor and the lubricating oil, the stator temperature in Fig.13 is obviously higher than that in Fig. 14. The comparison shows that the ends of the stator and rotor and heat source loss changes have a certain influence on the motor temperature analysis result.

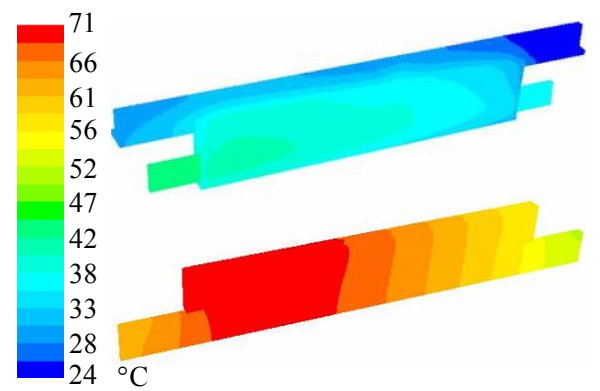

Figure 15. Temperature simulation without considering heat source loss change.

\section{Conclusions}

In this paper, the internal flow field, electromagnetic field and temperature field of Deep-water motor are numerically simulated. The following conclusions are drawn.

(1) In the air gap, the oil flow is dominated by swirling flow, and it velocity distribution decreases from the rotor side to the stator side. The pressure drop in the inner flow field decreases with the decrease of viscosity and inlet velocity. The friction loss of the rotor surface increases with the increase of the viscosity, but basically unchanged with the increase of the inlet velocity.

(2) After the start-up, the winding current fluctuates greatly and reaches a steady state after $75 \mathrm{~ms}$. The current decreases with the increase of rotor speed. After the motor is stable, the magnetic lines are evenly distributed on the stator yoke core. The iron loss density decreases with the increase of radial radius. The copper loss decreases with the increase of viscosity.

(3) Based on the results of internal flow field and magnetic field analysis, the law of motor temperature distribution is obtained. The maximum temperature of the motor appears at the air gap outlet. Compared with the temperature simulation considering only the air gap path, the stator temperature distribution law is obviously different. Compared with the result of temperature without considering heat source loss change, the maximum temperature of the motor decreased from $71^{\circ} \mathrm{C}$ to $49^{\circ} \mathrm{C}$

This research is sponsored by high technology ship research project of ministry of Industry and Information Technology, Grant No.2014020024.

\section{References}

1. Fu Xinghe, Lin Mingyao, Xu Da, et al. Computation and Analysis of 3D-Transient Temperature Field for a Permanent Magnet-Induction Hybrid Excitation Generator [J]. Transactions of China Electrotechnical Society, 28, 3 (2013): 107-113.

2. Chen Yiguang, Zheng Jun, Wei Juan, et al. Design of PMSM for Actuator and Its Temperature Field Analysis [J]. Transactions of China Electrotechnical Society, 30, 14 (2015): 94-99.

3. Qi Wenjuan, Zou Jiming, Hao Guiqing, et al. Thermal Analysis of Underwater Oil-filled BLDC Motors[C]. ICEMS, 24, 1 (2011): 1-4.

4. Li Ming, Tang Renyuan, Chen Lixiang. Screw Pump Permanent Magnet AC Servo Temperature of the Motor Calculation and Analysis[J]. Electrical Engineering, 9 (2012): 5-8.

5. Staton D.A, Cavagnino A. Convection Heat Transfer and Flow Calculations Suitable for Electric Machines Thermal Models[J]. IEEE Transactions on Industrial Electronics, 55, 10 (2008): 3509-3516.

6. Yujiao Z, Jiangjun R, Tao H, et al. Calculation of Temperature Rise in Air-cooled Induction Motors through 3-D Coupled Electromagnetic Fluiddynamical and Thermal Finite-element Analysis[J]. IEEE Transactions on Magnetics, 48, 2, (2012): 1047-1050.

7. Li Weili, Li Yong, Yang Xuefeng, et al. Temperature and Fluid Flow Field Calculation and Analysis of Stator End of Air Cooled Turbogenerator $[J]$. Proceedings of the CSEE, 29, 36, (2009): 80-87.

8. Zhang Yujiao, Ruan Jiangjun, Huang Tao, et al. Calculation of temperature rise in air-cooled induction motors through 3-D coupled electromagnetic fluid-dynamical and thermal finiteelement analysis[J]. IEEE Transactions on Magnetics, 48, 2, (2012): 1047-1050.

9. Yulei L. Calculation and Analysis of $3 D$ Temperature Field in Steady State of the Submersible Motor [C]. ICECE, (2010): 3427-3430. 
10. Vong P K, Rodger D. Coupled electromagneticthermal modeling of electrical achines[J]. IEEE Transactions on Magnetics, 39, 3, (2003): 16141617.

11. Meng Dawei, Liu Yulei, Zhang Qingjun, et al. Calculation and analysis of $3 D$ temperature of the submersible motor based on coupled field[J]. Electric Machines and Control, 14, 1, (2010): 52-55.

12. Meng Dawei, Liu Yu, Xu Yongming. Analysis and Calculation of 3D Temperature Field of Submersible
Motor Rotor $[J]$. Electric Machines and Control, 13, 3, (2009): 367-376.

13. Shehab Ahmed, Hamid A.Toliyat. Coupled Field Analysis Needs in the Design of Submersible Electric Motors[C]. IEEE Electric Ship Technologies Symposium. (2007): 231-237.

14. Zou Jibin, Qi Wenjuan, Xu Yongxiang, et al. Design of Deep Sea Oil-Filled Brushless DC Motors Considering the High Pressure Effect[J]. IEEE Transactions on Magnetics, 48, 11, (2012): 42204223. 